\title{
El inglés jurídico: análisis macroestructural de corpus digital de sentencias judiciales escocesas
}

\author{
Francisco Godoy Tena ${ }^{1}$ • (1) https://orcid.org/ 0000-0001-6353-6900
}

Universidad Autónoma de Madrid

Departamento de Filología Inglesa $\cdot$ Facultad de Filosofía y Letras · Despacho 201-VIBIS · Avenida Tomás y Valiente, $1 \cdot$ Campus de Cantoblanco $\cdot$ Universidad Autónoma de Madrid $\cdot 28049 \cdot$ Madrid $\cdot$ Spain

\section{RESUMEN}

La presente investigación se centra en el estudio de un corpus digital de 25 sentencias escocesas con el objetivo de establecer ciertos parámetros lingüísticos que puedan unificar la gran divergencia de sentencias judiciales de Escocia a través de una explicación de los epígrafes macroestructurales y del contenido de los mismos dentro de las sentencias escocesas.

Palabras clave: Escocia, sentencias judiciales, macroestructura, análisis de corpus.

\section{ABSTRACT}

The main purpose of this article is a research of a digital corpus of 25 Scottish judgments in order to establish certain macrostructural parameters. Such aim wants to establish certain linguistic parameters that may unify the great divergence of the Scottish judgments by means of the explanation of the macrostructural sections and their contents within the Scottish judgments.

Keywords: Scotland, judgments, macrostructure, corpus analysis.

\section{Inglés para fines específicos}

El estudio del "lenguaje para fines específicos" durante las últimas décadas es la consecuencia de una sociedad globalizada, en la que se han extendido relaciones comerciales más allá de los límites nacionales. Este es el caso de diversos ámbitos de la sociedad.

Originado en la década de 1950, momento en el que comienza a despuntar la economía mundial, el inglés para fines específicos ("English for Specific Purposes") cuenta con variedad de usos determinados. Con motivo de esta situación, se vio la necesidad de mejorar la comunicación especializada entre diferentes partes del mundo.

Dudley-Evans y Saint John (1998: 6-7) dividen el uso del "English for Specific Purposes" en dos grandes conjuntos: por una parte, el denominado "English for Academic Purposes" (EAP), en el que podemos distinguir "English for Science and Technology" (EST), aunque podemos considerar otras áreas, tales como "English for Medical Purposes" (EMP) y "English for Legal Purposes" (ELP). Por otro lado, existe el "English for Occupational Purposes" (EOP), y en él se engloba el lenguaje que no se encuentra enfocado para fines académicos, utilizado en situaciones profesionales. Este es el caso de la producción literaria, la científico-técnica 
y la jurídica, entre otras. De hecho, existe el denominado "inglés para fines jurídicos y judiciales", centrado en el uso especializado del lenguaje jurídico, objeto principal del presente estudio.

En este sentido, podemos mencionar a investigadores como Rey (1976), quien considera que tales son variantes léxicas del lenguaje general. A este respecto, se enmarcan las teorías de Sager, Dungworth y McDonald (1980), autores que defienden la condición especial de dichos lenguajes de especialidad, así como Harris (1968), Kittredge y Lehrberger (1982) o Lehrberger (1986), que consideran que los lenguajes de especialidad son sublenguajes, formados por unidades y construcciones sintácticas del lenguaje general.

En este punto, debemos mencionar opiniones divergentes, como la de Hoffmann (1979) y Lerat (1997), quienes puntualizan el hecho de que el lenguaje con fines jurídicos se encuentra separado del uso general del lenguaje, y que utiliza códigos lingüísticos con reglas diferentes de la lengua común.

Por otro lado, Hernando (2003) comparte de igual modo la importancia de la dimensión lingüística dentro del conocimiento y el quehacer humano, que aporta como resultado una mayor reflexividad en lo referente a la lengua y el papel del lenguaje especializado, dentro del cual se encuentra el lenguaje del derecho, del mismo modo que Alcaraz y Hughes (2009: 16). Más recientemente, podemos mencionar las investigaciones de Basturkmen (2010), García Laborda (2011), García Laborda y Litzler (2015) y García Laborda y Bejarano (2017), entre otros.

\section{Análisis de corpus y teoría macroestructural}

Dentro del panorama mencionado hasta ahora podemos incluir el análisis de corpus como método de primer orden en el inglés para fines específicos. Dicho estudio constituye un campo de investigación de gran trascendencia en diversas disciplinas. El presente trabajo se basa en la compilación de un corpus digital reciente de 25 sentencias de Escocia, distribuidas en función de los Courts y Tribunals dictadas y provenientes de diversos ámbitos y materias con el objetivo de poder establecer un patrón macroestructural aplicable a las sentencias escocesas.

La teoría de la macroestructura es el resultado de más de diez años de investigación por parte del lingüista van Dijk a fines de los años setenta y comienzo de los ochenta, basado en diversas disciplinas, como es el caso de la teoría literaria, la gramática textual, la teoría general de discurso, la pragmática y la psicología cognitiva. Sin embargo, debemos añadir igualmente la importancia de la estructura semántica, expresada por una serie o secuencia de frases (van Dijk, 1980b: 37; 1997: 90).

El análisis del corpus se centra en la teoría de la macroestructura de Van Dijk (1980a: v) quien define el concepto de "macroestructure" como "[a] higher-level semantic or conceptual structures that organize the "local' microstructures of discourse, interaction, and their cognitive processing".

La macroestructura textual de van Dijk se encuentra, por consiguiente, estrechamente ligada a la jerarquía semántica establecida en el texto. Dicho de otro modo, la macroestructura de van Dijk se establece a partir de unos valores estructurales, jerárquicamente establecidos, y también semánticos, coherentes dentro de cada unidad textual. 
La macroestructura ha sido considerada por numerosos investigadores a lo largo de los años (Kintsch y van Dijk, 1978; Kintsch y van Dijk, 1983; Freeman, 1991; Nwogu, 1997, García Izquierdo, 2005; Tabares, Ivanova y Krüger, 2008; Vázquez y del Árbol, 2008, 2014, 2016; Holl, 2011; Orts, 2016, entre otros).

La macroestructura de van Dijk organiza y establece, como hemos asegurado anteriormente, una estructura jerárquica de la información microestructural del texto y, por medio de su carga semántica, unifica todos los elementos microestructurales en un nivel más amplio, conformando así el nivel macroestructural.

Gracias a la organización macroestructural se puede establecer y planificar una organización estructural plena de significado, con una coherencia en su totalidad y que hace que sea identificada como una unidad diferente de otras. En este aspecto, podemos analizar discursos, conversaciones y, como en el caso que nos ocupa en el presente artículo, documentos jurídicos, entre los que las sentencias judiciales forman parte primordial de los mismos.

La macroestructura se puede describir como un conjunto de capas estructurales, gracias a las cuales podemos unificar un sistema coherente con el objetivo de desarrollar una historia en la mente del lector. En este sentido, el lector comienza a desarrollar una representación conceptual semántica, y que en su memoria denominamos macroestructura. Por consiguiente, la macroestructura forma parte de un resumen en un nivel abstracto de la estructura.

El presente estudio divide las sentencias escocesas en tres partes con el fin de realizar un análisis más claro y conciso: heading, main body y closure. Dicha división se ha establecido tras analizar la macroestructura de las sentencias judiciales provenientes del corpus analizado. Una vez realizado el estudio del corpus de las sentencias judiciales escocesas, se establecieron estas tres secciones supramacroestructurales:

- Heading: parte introductoria. Se incluyen las partes extralingüísticas (escudos), así como el nombre del Court, nombre de las partes, nombre del Sheriff, Lord o Lady, localidad, fecha y nombre de los letrados.

- Main body: cuerpo central. Parte fundamental de las sentencias judicial escocesas en las que se exponen el delito, las partes dispositivas de letrados, pruebas periciales y demás explicaciones básicas de la sentencia. Constituye la parte esencial de las sentencias judiciales y en la que se observa una mayor heterogeneidad macroestructural.

- Closure: parte final. Se incluye fundamentalmente la resolución final del Sheriff, Lord o Lady.

Pasamos a describir cada una de las secciones anteriormente mencionadas en las que se incluyen las macroestructuras y las denominaciones macroestructurales que componen dichos apartados.

\subsection{Heading}

El Heading o parte introductoria de las sentencias escocesas comienza con un escudo en la parte superior central - la única excepción es el caso del Sheriff Appeal Court-seguida del órgano judicial, junto con el área geográfica en la que se localiza el Court. No se observa en todas las sentencias: en el Court of Session (Inner y Outer House) no se menciona la localidad puesto que existe un único órgano.

A continuación, observamos el código de las sentencias del Sheriff Court, situados a derecha e izquierda de la parte superior de la misma. No obstante, los mencionados códigos no aparecen en todas ellas. 
Los nombres de los Sheriffs, Lords y Ladies se introducen en la parte superior central de las sentencias escocesas. Introducido por "Opinion of the Court. Delivered by", "Judgment of", o bien "Opinion of", sus nombres ocupan una posición predominante, siendo uno de los elementos compartidos en todas las sentencias de los órganos escoceses. Únicamente varía su posición en sentencias de órganos superiores, como en los Courts of Session (Inner House), Sheriff Appeal Courts y High Court of Justiciary, ya que figura localizado en la parte izquierda de las mismas.

Seguidamente, observamos la introducción de la legislación pertinente, observable en apartados independientes únicamente en las sentencias dictadas en los Sheriff Courts, Criminal Division.

El siguiente elemento macroestructural lo componen los nombres de las partes. Todas las sentencias de Escocia comparten dicha macroestructura en el "heading", localizado en la parte central del mismo. En ninguna de las sentencias de Escocia del corpus se han observado diferencias en cuanto a la fraseología utilizada en dicha macroestructura, siendo bastante homogéneas a este respecto.

Los nombres de los letrados se presentan en el siguiente apartado y prácticamente en todo el corpus analizado, a excepción de las sentencias dictadas en los Land Courts. Finalmente, el heading de las sentencias escocesas viene cerrado por la localidad y fecha. No encontramos citado el nombre de la localidad en diversas sentencias provenientes de órganos superiores, como el Court of Session, el Sheriff Appeal Court, y el High Court of Justiciary. La razón podría deberse a que el Court of Session y el Sheriff Appeal Court se encuentran en Edimburgo. En el caso del High Court of Justiciary, este cuenta con otras localizaciones, como Glasgow y Aberdeen.

\subsection{Main Body}

El main body de las sentencias escocesas constituye el apartado con el desarrollo macroestructural más extenso, puesto que la explicación tanto del Pursuer como del Defender en las sentencias escocesas constituye el marco contextual del litigio y, por ende, resulta la más extensa.

Las sentencias escocesas ofrecen un patrón macroestructural que presenta ciertas divergencias en el cuerpo central (main body) de las mismas. La mayor parte de ellas contienen, tras la introduction (o background), la macroestructura denominada submission, muy frecuente en las sentencias escocesas (frente al corpus de los otros sistemas jurídicos británicos).

El main body de las sentencias escocesas se presenta complejo, con numerosos epígrafes que aumentan las posibles denominaciones de las macroestructuras. No obstante, como se verá en la exposición y en la tabla resumida explicativa, las macroestructuras de las sentencias escocesas muestran cierto patrón concurrente en la mayor parte de ellas.

Primeramente, encontramos una Introduction, cuyo epígrafe es recurrente al comienzo de las sentencias de Escocia, llegando a eludirse en varios casos. A continuación, observamos el término NOTE (en mayúscula en las sentencias), común exclusivo en derecho escocés y utilizado especialmente en sentencias del Sheriff Court, con el objetivo de iniciar las explicaciones y el razonamiento propio de cada sentencia.

El segundo epígrafe esencial en las sentencias escocesas se denomina background que establece los hechos, motivos de petición de la parte actora, consideraciones previas del Judge (Sheriff, Lord o Lady), así como la 
descripción de la situación de ambas partes en litigio, y consideraciones previas a la vista. De igual modo, se vuelve a mencionar los nombres de los letrados de las partes, así como los posibles recursos a interponer por las partes.

No obstante, este apartado puede estar también destinado a la legislación pertinente para el caso. Su inclusión en un segundo lugar da primacía a la legislación, particularmente usual en los recursos. Así por ejemplo, observamos la macroestructura de la legislación tras la introduction en las siguientes sentencias escocesas:

- Sentencia [2016] SC DUN 15 del Sheriff Court (Criminal Division).

- Sentencia [2016] CSOH 51 del Court of Session (Outer House).

- Sentencia [2016] CSIH 12A del Court of Session (Inner House).

- Todas las sentencias del Land Court.

De igual modo, en esta segunda parte del main body escocés, observamos la inclusión de los razonamientos en los que se basan los recursos de apelación de las sentencias. En este sentido, el resto de los elementos informativos de las sentencias escocesas se desplazan hacia posiciones posteriores en las sentencias escocesas.

Los submissions constituyen la siguiente macroestructura de importancia en las sentencias escocesas. Se pueden considerar como las pretensiones y los argumentos de cada una de las partes de la litis, por medio de sus letrados. Estos últimos pueden igualmente exponer sus requerimientos e incluir los precedentes de sentencias anteriores, principalmente en recursos de apelación.

Su empleo en las sentencias de Escocia es muy evidente, siendo un epígrafe reiterado en todo el corpus escocés. A pesar de ello, no encontramos una homogeneidad en su denominación, apareciendo numerosos enunciados en la propia macroestructura, por lo que los mencionaremos detenidamente en la tabla destinada a tales efectos.

La siguiente sección macroestructural se denomina evidence $\mathrm{y}$, del mismo modo que la macroestructura submissions, aparece repetidamente en las sentencias de Escocia e incorpora en las sentencias analizadas los testimonios fundamentales de la misma.

Dichos testimonios (declaraciones) pasan a incluirse en un apartado algo más estable en cuanto a sus enunciados. No obstante, son varias las sentencias que desglosan dicha macroestructura en diversos subapartados que mejoren la comprensión de la misma. Podemos observar este caso en las siguientes sentencias:

- Sentencia [2016] CSOH 3 del Court of Session (Outer House). Se subdivide cada uno de los testimonios vertidos por los diversos especialistas en el tema del caso de la sentencia.

- Sentencia [2016] CSOH 47 del Court of Session (Outer House). Se desglosan los indicios a partir de los hechos, así como el testimonio aportado por parte de los peritos.

- Sentencia SLC/81/13 del Land Court. Se encuentra muy desglosada, ocupa más de 60 párrafos de la sentencia, subdivididos en función de las declaraciones de cada uno de los testigos de la misma.

El main body de las sentencias de Escocia finaliza con la macroestructura designada como discussion. Junto con la introduction, se la puede considerar como un elemento homogéneo en su denominación. Sin embargo, la macroestructura no es uniforme en cuanto a su distinción independiente dentro de la sentencia escocesa. 
La submission del Pursuer y del Defender y sus apartados constituyen el grueso de las sentencias escocesas, observándose en ella una mayor densidad macroestructural. Es en este punto de la macroestructura en el que las sentencias escocesas centralizan la información primordial de las mismas. La submission, clave en el main body de las sentencias escocesas, expone la descripción de ambas partes.

Por otra parte, resaltamos los informes periciales que se extienden en diversas sentencias escocesas, así como la parte expositiva de la cross-examination (contrainterrogatorio), otro de sus puntos esenciales del main body.

La legislación pertinente de las sentencias puede aparecer en cualquiera de los dos apartados de las macroestructuras de las sentencias escocesas. Por una parte, se incluye en la macroestructura submission y, por otro lado, en apartados diferenciados, denominados issues of facts, legislation o legal framework.

\subsection{Closure}

La parte final de la sentencia la constituye la decisión o fallo en el que se expone la resolución judicial, es decir, el cierre de las sentencias escocesas. La diversidad del main body en las sentencias escocesas parece finalmente unificarse en el closure. Gran parte de las sentencias del corpus se inician con conclusion, que introduce el fallo de la sentencia, de igual modo denominada decision, y que aporta ancillary arguments adicionales.

La parte final de las sentencias la constituyen otros comentarios adicionales, como es el caso del postscript, aunque no existe consistencia en la anexión de estos subapartados anejos a la conclusion. Sin embargo, debemos en este punto considerar la particularidad de las sentencias dictadas en el Land Court. Como comentamos anteriormente, las sentencias de dicho Court son diferenciables del resto y sucede lo propio en la macroestructura final de la misma.

En esta parte final del closure, las sentencias de los Land Courts incluyen los nombres de los letrados.

\section{Conclusiones macroestructurales}

Veamos algunas de las consideraciones de las sentencias escocesas en los siguientes puntos. Comenzaremos por los aspectos más generales:

- Macroestructura marcada en los encabezamientos de cada una de las partes de la sentencia escocesa.

Todas las sentencias escocesas muestran una macroestructura clara y visible. Solo observamos una carencia de macroestructura más homogénea en las sentencias de los Sheriff Appeal Courts. En estos casos, las sentencias no mantienen dichas características y centran su objetivo en la explicación precisa de los rasgos de la sentencia recurrida y los motivos y legislación pertinentes para su recurso.

- Análisis cronológico de las sentencias.

Además, las sentencias escocesas suelen aportar una descripción cronológica detallada de los hechos delictivos de las mismas. Este es el caso de la sentencia [2016] HCJAC HCA/2014/004974/XC del High Court of Justiciary, en la que observamos la enumeración cronológica en su párrafo número 3.

De igual modo, observamos una explicación cronológica en los siguientes ejemplos: 
- Sentencia [2016] CSOH 47 del Court of Session (Outer House).

- Sentencia [2016] CSOH 51 del Court of Session (Outer House).

- Sentencia [2016] SC EDIN 7 del Sheriff Court (Criminal Division).

- Macroestructura detallada en las sentencias escocesas.

Las sentencias escocesas muestran una macroestructura más detallada, con unos patrones estructurales ligeramente más estables en el marco de las sentencias británicas. Así, mencionamos a continuación algunas sentencias en las que observamos una macroestructura más asentada:

- Sentencias [2016] EDIN SC 6 y 2015SCGLA63 del Sheriff Court (Civil Division)

- Todas las sentencias del Land Court.

- Importancia de los submissions y los testimonios (evidence) en las sentencias escocesas.

Hemos de destacar la importancia de los submissions, común a las sentencias analizadas del corpus. Igualmente, señalamos las pruebas periciales (evidence o productions), esenciales también en las sentencias estudiadas. Dentro de los evidence cabe mencionar, a modo de ejemplo, las sentencias del High Court of Justiciary, en donde se consideran los testimonios o evidencias de los siguientes expertos: facultativos biomédicos y forenses, ingenieros y autoridades policiales.

- Macroestructuras extensas en las sentencias escocesas.

Gran parte de las sentencias dictadas en los Sheriff Courts poseen tanto una extensión como un desarrollo macroestructural complejo. Por el contrario, las sentencias de los Sheriff Appeal Courts muestran, en general, una extensión macroestructural más reducida, ya que se condensa en pocos párrafos la información de todos los hechos de la sentencia en cuestión.

- Macroestructuras especiales de las sentencias escocesas.

Dentro del corpus de las sentencias escocesas vamos a detenernos en la macroestructura de dos órganos judiciales escoceses en los que conviene mencionar, a modo de conclusión, ciertos rasgos especialmente distintivos del resto de las sentencias escocesas.

En primer lugar, citaremos las sentencias emanadas del High Court of Justiciary, en el que la macroestructura difiere con respecto al resto de las sentencias.

En segundo lugar, mencionamos igualmente las sentencias de los Land Courts escoceses. Podemos considerarlo un órgano judicial particular escocés, y en cuya sentencia vemos numerosos aspectos que conviene resaltar. De hecho, podemos señalar la densidad de las sentencias del Land Court. La explicación del hecho juzgado en cada una de las sentencias se presenta muy detallada y con una carga sintáctica extremadamente densa, con oraciones complejas y muy detalladas en su heading.

Las sentencias del Land Court comienzan con un orden macroestructural claramente diferenciador de otras sentencias escocesas. La falta de claridad de dichas sentencias por su densidad macroestructural, sobre todo en su heading, se suple por la enumeración de palabras claves (keywords) que nos ponen en antecedentes frente a cada sentencia. 
En el siguiente punto, y a modo de conclusión, se va a exponer una tabla que resuma las macroestructuras de las sentencias analizadas, con el objetivo de sintetizar sus aspectos clave. En ella se incluyen las partes macroestructurales, lingüísticas y extralingüísticas reflejadas en la posición en las que aparecen en las sentencias judiciales analizadas.

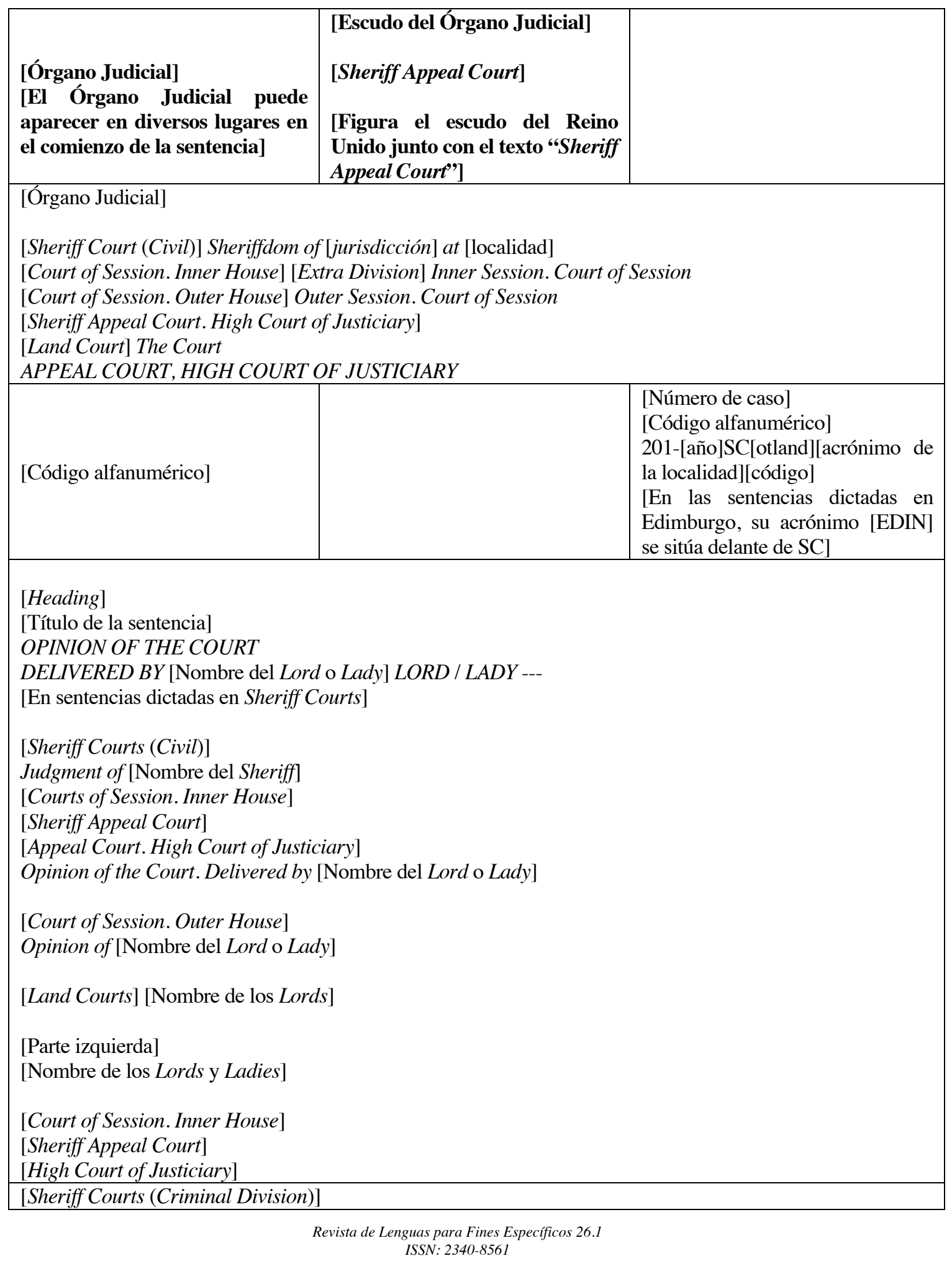




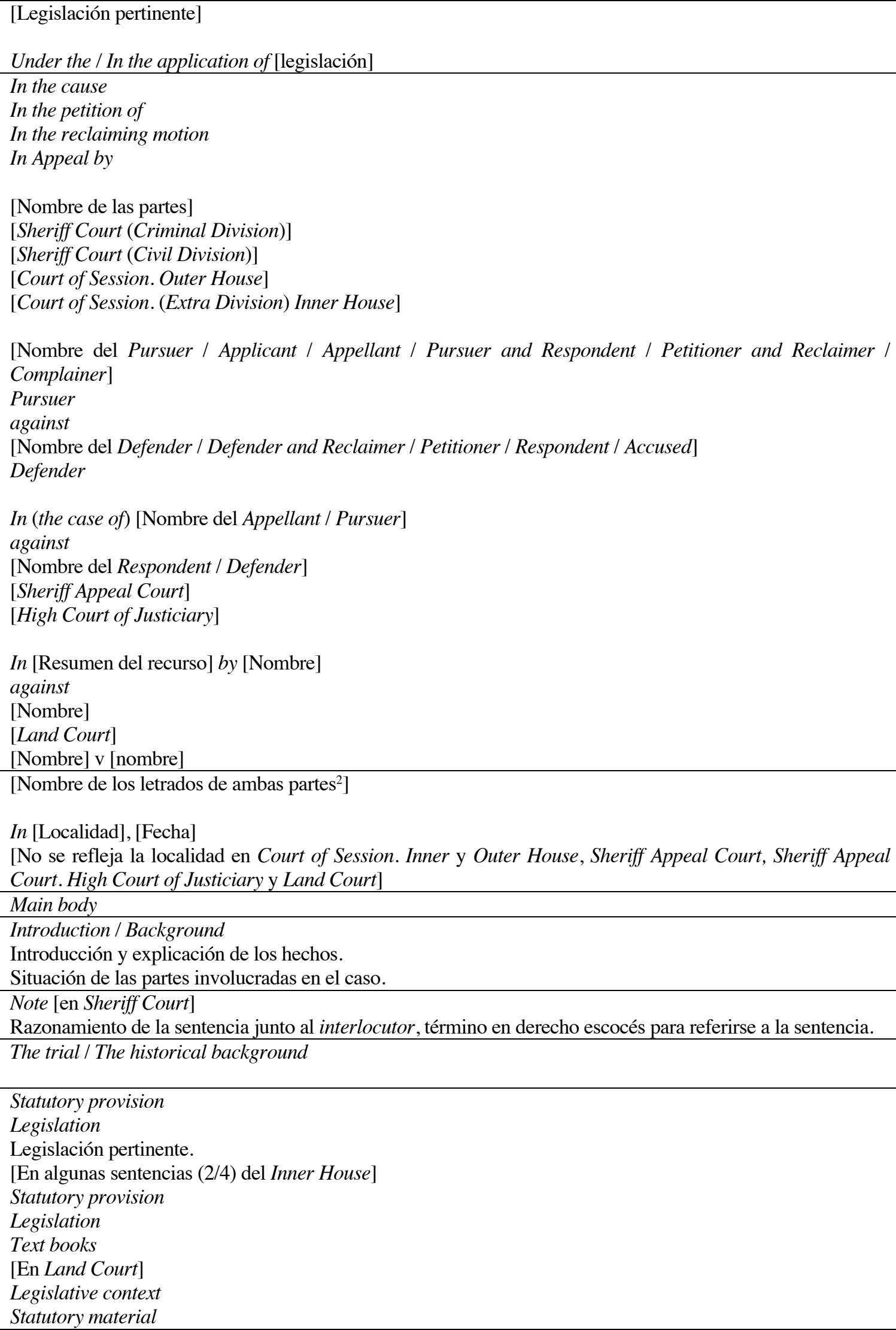

[Nombre del Pursuer / Applicant / Appellant / Pursuer and Respondent / Petitioner and Reclaimer / Complainer]

Pursuer

against

[Nombre del Defender / Defender and Reclaimer / Petitioner / Respondent / Accused]

Defender

In (the case of) [Nombre del Appellant / Pursuer]

against

[Nombre del Respondent / Defender]

[Sheriff Appeal Court]

[High Court of Justiciary]

In [Resumen del recurso] by [Nombre]

against

[Nombre]

[Land Court]

[Nombre] v [nombre]

[Nombre de los letrados de ambas partes ${ }^{2}$ ]

In [Localidad], [Fecha]

[No se refleja la localidad en Court of Session. Inner y Outer House, Sheriff Appeal Court, Sheriff Appeal Court. High Court of Justiciary y Land Court]

Main body

Introduction / Background

Introducción y explicación de los hechos.

Situación de las partes involucradas en el caso.

Note [en Sheriff Court]

Razonamiento de la sentencia junto al interlocutor, término en derecho escocés para referirse a la sentencia.

The trial / The historical background

Statutory provision

Legislation

Legislación pertinente.

[En algunas sentencias (2/4) del Inner House]

Statutory provision

Legislation

Text books

[En Land Court]

Legislative context

Statutory material

2 En todas las sentencias del corpus, excepto en todas las sentencias del Land Court.

Revista de Lenguas para Fines Específicos 26.1 ISSN: 2340-8561 


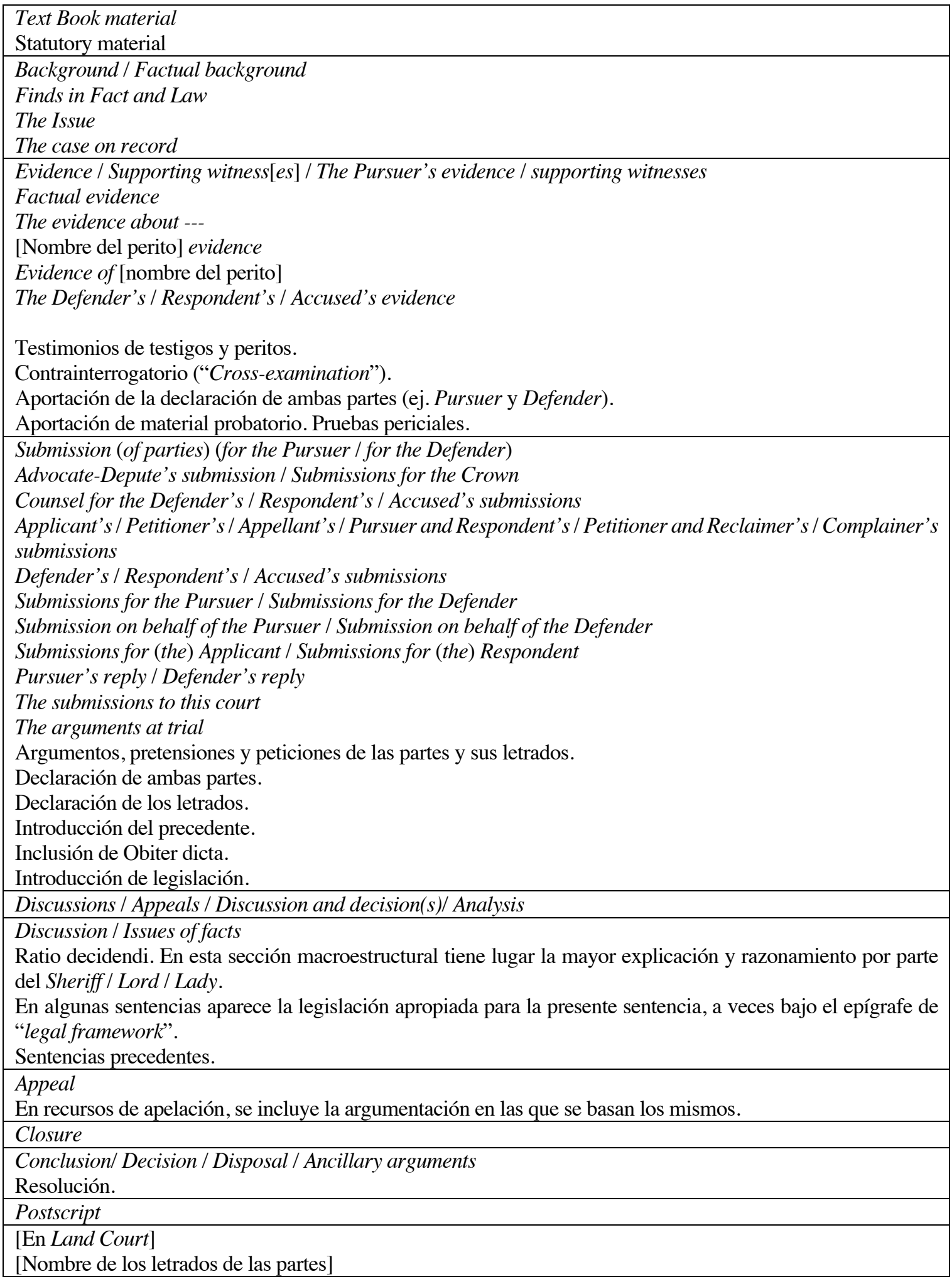

Table 1. Propuesta resumida de análisis macroestructural de sentencias escocesas. 


\section{About the author}

Francisco Godoy Tena, Profesor en el Grado de Traducción e Interpretación de la Universidad Autónoma de Madrid durante 9 años. Doctorado Cum Laude en junio de 2017. Docencia en Traducción Jurídica-Judicial inversa (español-inglés), Traducción Científico-Técnica directa (inglés-español) y Traducción General directa e inversa (español-inglés-español), entre otras. Ha publicado diversos artículos de investigación en Traducción Jurídico-Judicial. De igual modo, ha presentado varias ponencias en Congresos realizados en Alcalá de Henares, Vigo, Madrid y Olomouc, entre otros. En la actualidad es miembro del Grupo de Investigación UAM TransPoliLex ("Léxico, Discurso y Traducción Jurídico-Policial en Contextos Multilingües").

\section{References}

Alcaraz Varó, E. y Hughes, B. (2009). El español jurídico. Barcelona: Ariel.

AA. VV., 2018: "Sentences and Appeals". Scottish Sentencing Council. <https://www.scottishsentencingcouncil.org.uk/aboutsentencing/sentences-and-appeals $>[27 / 11 / 2018]$.

AA.VV. Scottish Courts and Tribunals. <[https://www.scotcourts.gov.uk/search-judgments/about-judgments]>[27/11/2018].

Baker, M. (1996). Corpus-based translation-studies: The challenges that lie ahead. En Harold soMers (Ed.): Terminology, LSP, and Translation: Studies in Language Engineering in Honor of Juan C. Sager (pp. 175-186). Amsterdam/Philadelphia: John Benjamins.

Basturkmen, H. (2010). Developing Courses in English for Specific Purposes. London: Palgrave McMillan.

Busby, N., Clark, B. y Paisley, R. (2000). Scots Law: A Student Guide. Edinburgh: T\&T Clark.

Clark, B. y Keegan, G. (2012). Scottish Legal System Essential: Edinburgh: Edinburgh University Press.

Crossan, S. (2017). Introductory Scots Law Third Edition: Theory and Practice. London: Hodder Education.

Dudley-Evans, T. y St. John, M. J. (1998). Developments in English for Specific Purposes. Cambridge: Cambridge University Press.

Ferguson, P.R. y McDiarmid, C. (2015). Scots Criminal Law. A Critical Analysis. Edinburgh: Edinburgh University Press.

Freeman, J.B. (1991). Dialectics and the Macrostructure of Arguments: A Theory of Argument Structure. New York: Foris Publications.

García Izquierdo, I. (2005). El género textual y la traducción. Bern: Peter Lang.

García Laborda, J. (2011). Revisiting materials for teaching Languages for Specific Purposes. 3L The Southeast Asian Journal of English Language Studies, 17 (1), 102-112.

García Laborda, J. y Bejarano, L. G. (2017). ¿Hablamos de lo mismo? Estudio contrastivo de programas de lenguas para fines específicos en dos universidades de Estados Unidos y España. Revista de Lenguas para Fines Específicos 23.1 (2017), 63-82.

García Laborda, J., y Litzler, M.F. (2015). Current approaches in teaching English for Specific Purposes. Onomázein, 31 , 38-51.

Harris, Z. (1968). Mathematical Structures of Language. New York: John Wiley and Sons.

Harvey-Clark, S. (2009). "SPICe The Scottish Civil Court System”. The Scottish Parliament. (9/52). <http://www.scottish.parliament.uk/Research\%20briefings\%20and\%20fact\%20sheets/SB09-52.pdf>[27/11/2018].

--- 2014: SPICe The Scottish Civil Court System. The Scottish Parliament. $<$ http://www.parliament.scot/ResearchBriefingsAndFactsheets/S4/SB_14-15.pdf>[27/11/2018].

Hernando Cuadrado, L. A. (2003). El lenguaje jurídico. Madrid: Verbum.

Hoffmann, L. (1979). Towards a Theory of LSP. Elements of a Methodology of LSP Analysis. Fachsprache 1. 1-2.

Holl, I. (2011). Die Konstrastive Textsorttenanalyse als Vostufe zur Übersetzung von Rechtstexten: Deutsche und Spanische Scheidungsurteile im Vergleich. Revista de Lingüística y Lenguas Aplicadas. (6), 195-208. 
Kintsch, W. y Van Dijk, T.A. (1978). Towards a Model of Text Comprehension and Production. Psychological Review (85) 5, 363394.

--- (1983). Strategies of Discourse Comprehension. London: Academic Press.

Kiralfy, A. y MacQueen, H. L. (2016). New Perspective in Scottish Legal History. London: Routledge.

Kittredge, R. y Lehrberger, J. (1982). Sublanguages. Studies of Language in Restricted Semantic Domains. Berlin: Walter de Gruyter. Lerat, P. (1997). Las lenguas especializadas. Barcelona: Ariel.

MacQueen, H. L., (1993). Studying Scots Law. Edinburgh: Butterworths Law.

Himsworth, C. y O’Neill, C.M. (2015). Scotland's Constitution: Law and Practice. London: Bloomsbury Professional.

Lehrberger, J. (1986). Sublanguage Analysis. En Grishman, R. y Kittredge, R. (Eds.). Analyzing Language in Restricted Domains. (Pp. 19-38). Hillsdale: Lawrence Erlbaum Associates.

Nwogu, K. (1997). The Medical Research Paper: Structure and Functions. English for Specific Purposes 16 (2), 119-138.

Ormerod, D. (2008). Smith and Hogan Criminal Law. Oxford: Oxford University Press.

Orts, M.A. (2016). An Approach to the Study of Public Law: A Pragmatic-discursive Analysis of Judicial Decisions in Spanish and English. Fachsprache, 1(2), 67-83.

Padfield, N. (2012). Criminal Law. Oxford: Oxford University Press.

Rey, A. (1976). Néologie en marche, série b: langues de spécialité 2. Québec: Gobierno.

Sager, J. C., Dungworth, D. y McDonald, P. F. (1980). English Special Languages: Principles and Practice in Science and Technology. Wiesbaden: O. Brandstetter.

Tabares, E., Ivanova, V. y Krüger, E. (2008). Análisis lingüístico contrastivo de textos especializados en español y alemán. Berlín: Frank \& Timme GmbH.

Van Dijk, T.A. (1980a). Macrostructures: An Interdisciplinary Study of Global Structures in Discourse, Interaction, and Cognition. Hillsdale: Lawrence Erlbaum.

--- (1980b). Texto y contexto. Madrid: Cátedra.

Vázquez y del Árbol, E. (2008). La traducción (inglés-español) de testamentos británicos y documentos relacionados. De la teoría a la práctica. Granada: Universidad.

--- (2014). Los actos procesales: estudio macroestructural contrastivo (inglés-español) aplicado a la traducción. Fachsprache 3-4, 179204.

--- (2016). Traducción judicial y policial (inglés <> español) y Derecho Comparado. Madrid: Dykinson. 